\title{
Classification behavior and measures of intelligence: Dimensional identity versus overall similarity
}

\author{
THOMAS B. WARD, BRIAN H. STAGNER, JAMES G. SCOTT, \\ SUSAN T. MARCUS-MENDOZA, and DARRELL TURNER \\ Texas A\&M University, College Station, Texas
}

\begin{abstract}
Individuals tend to adopt either analytic or holistic modes of categorizing objects. In two studies, we examined the relation between these categorization tendencies and cognitive abilities as measured by standard psychometric instruments. The participants in both studies were pretested with a restricted classification task in which it was possible for them to classify simple stimuli by dimensional identity or overall similarity. Those making a large number of either type of categorization were then tested with subtests of the WAIS-R and with the Raven's progressive matrices. Across both studies, the analytic individuals (many dimensional identity classifications) scored higher than the holistic individuals (many overall similarity classifications) on some but not all of the subtests. The results are consistent with the idea that holistic modes of categorization may be more "primitive" than analytic modes. The findings are discussed in terms of the association between categorization mode and either general or specific cognitive abilities.
\end{abstract}

A basic human tendency is to categorize objects rather than to treat each object as a unique entity. Since any given set of objects can be partitioned into smaller subgroups in a variety of ways, the rules by which such categorizations are made have been the focus of a considerable amount of research. Of the possible rules that could be used, two in particular appear to predominate, at least with simple multidimensional stimuli. These are dimensional identity and overall similarity. According to the first of these rules, objects would be categorized together if they matched exactly along some particular component dimension or attribute, even though they might differ considerably along other dimensions or in other attributes. For example, all squares, all red objects, or all creatures with two legs may be designated as belonging in the same group regardless of differences in any of their other attributes. According to the second rule, objects would be categorized together if they were perceived as similar to one another in an overall sense even though they might not be identical along any of their component dimensions. Natural categories, with their family-resemblance structure, have often been cited as examples of this type of categorization.

A number of factors appear to determine whether dimensional identity or overall similarity partitionings will be observed. These include stimulus integrality (Garner, 1974), age (L. Smith \& Kemler, 1977), task conditions (J. Smith \& Kemler Nelson, 1984; Ward, 1983), and in-

\footnotetext{
The results of Study 1 were presented at the meeting of the American Psychological Association, New York, August 1987. This work was supported by Grant MH 39369 from the National Institute of Mental Health to Thomas B. Ward. Address all correspondence to Thomas Ward or Brian Stagner, Department of Psychology, Texas A\&M University, College Station, TX 77843.
}

dividual differences (J. Smith \& Baron, 1981; Ward, 1983). In the present two studies, individual differences among adult observers are of most interest. More specifically, in the present studies we were concerned with asking what other aspects of cognitive functioning might be related to the tendency of adult observers to classify by dimensional identity as opposed to overall similarity.

There is some evidence that individual differences in the mode of classification exhibited by adults may be associated primarily with stylistic preferences for particular types of rules rather than with differences in cognitive abilities. For example, J. Smith and Baron (1981) found that a composite score computed from performance on the Raven's progressive matrices and SAT scores did not correlate significantly with the number of dimensional identity classifications made by college students. On the other hand, Kemler (1982) and Burns (1986) observed more overall similarity and fewer dimensional identity classifications on the part of retarded individuals than on the part of their nonretarded peers. In addition, Ward (1985) and Ward, Foley, and Cole (1986) found that embedded figures test performance (generally thought to be a measure of ability; see e.g., Kogan, 1973) was significantly related to the tendency to make dimensional identity classifications.

The above findings indicate that two factors may influence the extent to which links between abilities and classification behavior are observed. The first factor is the range of differences on either the ability measure or the classification behavior measure. Kemler's (1982) retarded and nonretarded subjects, for example, exhibited large IQ differences, whereas J. Smith and Baron's (1981) subjects were all college students, who would be expected to show a smaller range of both IQ and classification responses. 
In addition, even with a full range from primarily dimensional identity responses to primarily overall similarity responses, the latter pattern is uncommon among college students: there may not have been enough individuals at that extreme for significant relations to have been found. The second factor is the specificity of the ability being measured. The SAT scores used by J. Smith and Baron can be viewed as a more general measure of ability, whereas the embedded figures test performance used by Ward (1985; Ward et al., 1986) can be more reasonably thought of as a more specific measure of visuospatial ability.

The present studies were designed in order to examine further the links between individuals' modes of categorization and their measured cognitive abilities. A wide range of classification performance was examined along with a number of different general and specific ability measures. The wide range of classification behavior was obtained by prescreening participants in a restricted classification task and selecting only those who exhibited the greatest number of either dimensional identity or overall similarity classifications. The ability measures used were subtests of commonly used intelligence tests. The studies are part of a larger effort to determine whether differences in classification behavior, as measured by laboratory tasks with simple stimuli, can be meaningfully related to performance on a variety of measures that are used in applied settings.

\section{STUDY 1}

The first study involved the Vocabulary and Block Design subtests of the Wechsler Adult Intelligence ScalesRevised (WAIS-R; Wechsler, 1981) and the Raven's Progressive Matrices (Raven, Court, \& Raven, 1983). Previous work involving embedded figures test performance indicates that dimensional identity versus overall similarity classification performance would be most likely to predict differences in performance on tasks that tap visuospatial organization (Ward, 1985) and would presumably be less predictive of differences in performance on tasks that rely on verbal ability. Thus it was predicted that the strongest relation to classification behavior would be found in performance on the Block Design test and the weakest would be found in performance on the Vocabulary subtest, because these two subscales are recognized, respectively, as the best indicators of visuospatial and verbal ability (Lezak, 1983). Since the Raven's test is supposed to reflect both verbal and visuospatial ability (Lezak, 1983), it was unclear to what degree classification behavior would predict performance on this test. However, results such as those reported by J. Smith and Baron (1981) for the Raven's and SAT combined indicate that a strong relation might not be observed.

\section{Method}

Subjects. The participants were 73 undergraduate students enrolled in introductory psychology classes at Texas A\&M University. This group was selected from a larger group of 1155 in- dividuals, on the basis of their performance on a restricted classification pretest as described below. Of those who were contacted after their pretest performance, 30 individuals with strong overall similarity patterns and 43 individuals with strong dimensional identity patterns agreed to be tested on the WAIS-R and the Raven's.

Stimuli. The two sets of stimuli for the restricted classification task were circles that varied in area and orientation of the radius within the circle, and single lines of dots that varied in length and density. The circles were chosen from the set defined by the possible combinations of areas of $4.5,6.4,9.0,12.8,18.8$, and 25.5 $\mathrm{cm}^{2}$ and radius orientations of $5^{\circ}, 35^{\circ}, 65^{\circ}, 95^{\circ}, 125^{\circ}$, and $155^{\circ}$ from vertical. The lines of dots were chosen from the set defined by the possible combinations of lengths of $1,1.5,2,3,4,6$, and $8 \mathrm{~cm}$ and densities of $1.5,1, .75, .50, .25$, and $.125 \mathrm{~cm}$ of interdot distance. Both circles and dots were black figures on white backgrounds, photographed for presentation as slides.

For both the circles and the dots, 24 triads of stimuli were constructed. The triads can be conceptualized in terms of the three possible subsets that can be created by selecting two out of the three items to go together. Within each triad two of the items were identical on one dimension and very different on the other dimension (e.g., by three or more levels). These items would be grouped together by an individual who was responsive to dimensional identity. In addition, two of the items in each triad were highly similar in overall appearance, although they differed slightly on both dimensions (e.g., by one level on each of the two dimensions). An individual who was responsive to overall similarity would choose to group these items together. The remaining pairing for each triad involved items that were neither identical on one dimension nor highly similar overall. This last type of grouping is rarely observed among adult participants. For the circles, 12 of the 24 triads had two items that were identical in area and 12 had two items that were identical in terms of radius orientation. For the dots, 12 triads had two items that were identical in length and 12 had two items that were identical in density. Because of the structure of the triads it is possible to determine whether individuals tend to classify stimuli either analytically or holistically (i.e., in terms of identity along their component dimensions, as opposed to overall appearance; see Garner, 1974). Such restricted classification triads have been used extensively in order to examine dimensional identity versus overall similarity classifications (e.g., Shepp, Burns, \& McDonough, 1980; L. Smith \& Kemler, 1977; Ward et al., 1986). In addition, previous research has revealed strong individual differences across the two sets of materials. For example, Ward (1983) found a significant correlation of .50 between dimensional identity responses and a significant correlation of .54 between the similarity responses to the two sets. In addition, Ward et al. (1986) reported a significant correlation of .64 between dimensional responses and a significant correlation between similarity responses to the two sets. Thus, performance on the two sets combined can be used to select individuals with a general tendency to categorize multidimensional stimuli either analytically or holistically.

The other stimuli used were the Vocabulary and Block Design subtests of the WAIS-R, the 60-item Raven's Standard Progressive Matrices, and a set of four unsolvable anagrams.

Procedure. All individuals pretested on the restricted classification task were shown the circle and dot triads one at a time and were asked to pick, for each triad, the two items out of the three that went together best. They were given 5 sec to make their choice for each triad. Participants judged all 24 triads for the circle materials and then judged all 24 for the dot materials. The number of analytic (dimensional identity) and holistic (overall similarity) responses was tabulated for each participant. Individuals who made dimensional identity classifications for at least two-thirds (32 out of 48) of the triads across both sets of materials were identified as analytic responders, and those who made overall similarity classifications for at least $\mathbf{3 0}$ out of the $\mathbf{4 8}$ triads were identified as holistic 
responders. In addition, to ensure that assignment to the analytic or holistic group was based on a general response tendency across both sets of materials, a further constraint was that the majority of the person's responses on each of the sets separately had to be either dimensional identity or overall similarity types. Thus, for example, an individual who made 24 dimensional responses to one set and 8 to the other set would not be identified as analytic, despite the fact that the person's total number of dimensional responses was 32 out of 48 .

The analytic and holistic responders were contacted for further testing. Those who agreed to participate further were tested in two subsequent sessions. In one session, the Raven's test was administered according to standard procedures. In the other session, the Vocabulary and Block design subtest were administered according to standard procedures (Wechsler, 1981). A measure of persistence was also used, involving the amount of time that an individual would continue trying to solve a set of four unsolvable anagrams before giving up. Participants were given a sheet of paper containing four unsolvable anagrams and were told to try to unscramble each of the four sets of letters to form a word. They were told to take as much time as they needed and to contact the experimenter if they were unable to solve the anagrams. The experimenter then left the room and returned at the request of the subject or after a maximum of $15 \mathrm{~min}$.

\section{Results and Discussion}

Separate analyses of variance were conducted on the Raven's, Vocabulary, Block Design, and persistence measures using group (analytic vs. holistic) and sex as between-subjects variables. There were no significant main effects or interactions involving the variable of sex. In contrast, the group variable revealed important differences. The analytic individuals performed significantly better on Block Design [mean $=117.3$ vs. $111.3 ; F(1,69)$ $=5.64, p<.05]$, and were significantly more persistent $[$ mean $=290.5$ vs. $467.6 \mathrm{sec} ; F(1,69)=9.27$, $p<.01]$, than the holistic individuals. The groups did not differ significantly on the Raven's $[F(1,69)=1.68$, $p>.19$ ], or on Vocabulary $(F<1)$.

The effect for Block Design is what would be expected if analytic individuals are viewed as having greater visuospatial ability than holistic individuals. In addition, the effect for persistence is consistent with previous descriptions of fast holistic and slow analytic processors (see e.g., Cooper, 1976; Cooper \& Podgorny, 1976; Ward, 1983).

Since the analytic individuals were more persistent than the holistic individuals, it might be argued that the differences in Block Design were determined by that factor rather than by ability. However, an analysis of covariance revealed that the group difference in Block Design remained even when persistence, Raven's performance, Vocabulary performance, and sex all were used as covariates $[F(1,65)=4.21, p<.05]$. The results confirm the expectation that individual differences in analytic and holistic processing are related in meaningful ways to performance on standardized psychological tests. They also indicate that further investigations into the relation between basic cognitive processes and performance on measures of ability used in applied settings are justified and can lead to greater understanding of each of those domains.

\section{STUDY 2}

The results of our first study indicate that classification behavior is related to some measures of ability and not to others. Specifically, it appears that a good performance on a measure of visuospatial ability is associated with high levels of dimensional identity classification. The result is consistent with previous observations that embedded figures test performance is associated with high levels of this type of classification (Ward et al., 1986). The second study was conducted to examine the generality of the link between classification behavior and measures of particular abilities. As in Study 1, participants were prescreened and divided into groups of highly analytic and highly holistic individuals. These extreme groups were then tested with all 11 subtests of the WAIS-R. If classification behavior is linked to visuospatial ability rather than overall ability, then only performance on the certain subtests should be significantly related to the tendency to make dimensional identity versus overall similarity classifications. Specifically, there appears to be a factor of perceptual organization with large weights on both the Block Design and Object Assembly subtests (see e.g., Anastasi, 1982). It might be expected that analytic and holistic classifiers would differ on this factor. In contrast, there also appears to be a factor of verbal comprehension with weights on Vocabulary, Information, Comprehension, and Similarities subtests (Anastasi, 1982). If the link between classification behavior and ability is specific to visual and spatial abilities, then it might be expected that there would be no differences between analytic and holistic individuals on this verbal comprehension factor.

\section{Method}

Subjects. The participants were 59 undergraduates enrolled in introductory psychology classes. As in Study 1, this group was chosen on the basis of restricted classification pretest performance from a group of approximately 900 individuals. Twenty-three holistic and 36 analytic individuals agreed to participate.

Stimuli and Procedures. The stimuli and procedures for the prescreening classification task as well as the selection criteria for analytic and holistic participants were the same as described for Study 1. Participants who met the selection criteria and who agreed to participate further were administered all 11 subtests of the WAIS-R in a single individual testing session.

\section{Results and Discussion}

The mean scaled scores on each subtest as well as the Verbal, Performance, and Full Scale IQ scores are shown in Table 1 separately for the analytic and holistic individuals. The table also contains $F$ and $p$ values for the group comparisons. The differences between the analytic and holistic groups on the 11 subscales were assessed using a multivariate analysis of variance procedure. The results of that analysis confirm two of the important findings of the first study. That is, the analytic individuals performed significantly better than the holistic individuals on the Block Design subtest, but the groups did not differ on the Vocabulary subtest. In addition, the analytic in- 
Table 1

Mean Scaled Scores on 11 WAIS-R Subtests, and on Verbal, Performance, and Full Scale IQ, for Analytic and Holistic Responders

\begin{tabular}{lccccc}
\hline & \multicolumn{2}{c}{ Group } & & \multicolumn{2}{c}{$\begin{array}{c}\text { Group } \\
\text { Comparison } \\
\text { Value }\end{array}$} \\
\cline { 2 - 3 } \cline { 5 - 6 } \multicolumn{1}{c}{ Score Type } & Analytic & Holistic & & $F$ & $p$ \\
\hline Information & 10.19 & 10.56 & & 0.49 & n.s. \\
Digit Span & 11.91 & 10.74 & & 2.78 & n.s. \\
Vocabulary & 10.77 & 10.65 & & 0.08 & n.s. \\
Arithmetic & 12.47 & 10.82 & & 7.27 & .01 \\
Comprehension & 12.77 & 10.87 & & 8.65 & .01 \\
Similarities & 11.75 & 10.65 & & 2.98 & n.s. \\
Picture Comp. & 11.02 & 9.87 & & 4.27 & .05 \\
Picture Arr. & 11.91 & 11.09 & & 1.41 & n.s. \\
Block Design & 14.41 & 13.08 & & 4.01 & .05 \\
Object Assembly & 12.41 & 11.26 & & 2.03 & n.s. \\
Digit Symbol & 12.77 & 12.52 & & 0.25 & n.s. \\
Verbal IQ & 117.0 & 110.6 & & 5.16 & .05 \\
Performance IQ & 121.8 & 113.9 & & 5.49 & .05 \\
Full Scale IQ & 121.4 & 113.6 & 6.90 & .05 \\
\hline
\end{tabular}

dividuals had significantly higher scores than the holistic individuals on Arithmetic Reasoning, Comprehension, and Picture Completion, as well as on Verbal, Performance, and Full Scale IQ. These last three differences, however, appear to be mediated by the reliable differences on the four subtests. When the scores from the Block Design, Arithmetic Reasoning, Comprehension, and Picture Completion subtests are removed, a composite score constructed by averaging the scaled scores from the remaining subtests no longer reveals a significant difference between the analytic and holistic participants $[F(1,57)=$ $3.06, p>.05$ ].

An additional set of analyses was conducted to examine differences between analytic and holistic responders on composite scores for the verbal comprehension and perceptual organization factors, as well as for a third factor referred to as memory. All of these factors have consistently emerged from factor analyses of WAIS performance (Anastasi, 1982). The verbal composite, which was the average of the scaled scores on Vocabulary, Information, Comprehension, and Similarities, did not reveal a significant difference between the groups $[F(1,57)=2.83$, $p>.05]$. The perceptual organization composite, which was the average of the scaled scores for Block Design and Object Assembly, fell just short of indicating a significant advantage for the analytic individuals over the holistic individuals $[F(1,57)=3.81, p=.056]$. Finally, the memory factor, which was a composite of the scaled scores for Arithmetic Reasoning and Digit Span, revealed a significant advantage for the analytic individuals $[F(1,57)=7.05, p<.05]$.

\section{GENERAL DISCUSSION}

The results of both studies confirm that differences in individuals' preferred modes of categorizing objects are related to differences in abilities as measured by some sub- tests of standardized intelligence tests. Combined with previous failures to find differences (J. Smith \& Baron, 1981) and previous successes (Kemler, 1982), the results suggest that a wide range of differences either in classification behavior (present studies) or in measured intelligence (Kemler, 1982) may be necessary to find significant links between abilities and modes of classification. The subjects in the present study were selected specifically because of the large discrepancy in their modes of classification; they represent the extremes on the distribution of modes of classification behavior.

It is interesting that, with the exception of the scores on the Information subtest, all of the differences between the groups favor the analytic individuals (see Table 1). The findings, then, are consistent with a wide variety of other findings, which indicate that holistic modes of categorization are more primitive or less well-developed than analytic modes. Previous findings include the observation that holistic modes of categorization are more prevalent in young children (Shepp et al., 1980; L. Smith \& Kemler, 1977; Ward, 1980), in adults who are forced to respond under time pressure (J. Smith \& Kemler Nelson, 1984; Ward, 1983) or cognitive load (J. Smith \& Kemler Nelson, 1984), and in adults who have generally fast response tempos (Ward, 1983; Ward et al., 1986). To this list of findings we now add the observation that, even among an otherwise fairly homogeneous group of college freshmen, lower scores on a standardized psychometric instrument are associated with a tendency to classify by overall similarity.

A question that arises from the results is whether the differences between analytic and holistic categorizers are indicative of differences in a general intelligence factor or differences in more specialized skills such as visuospatial analysis. There is some evidence to support each of these positions.

On the side of general differences, there were significant differences between the analytic and holistic groups on Verbal, Performance, and Full Scale IQ. However, those differences appeared to be mediated by large differences on a few of the subtests. Thus it is not clear whether the Full Scale IQ difference should be interpreted as indicating differences in a general intelligence factor.

Analyses of the scores in terms of the types of factors normally observed for WAIS-R subtest performance also yield a mixed picture. On the one hand, the subtests that revealed significant differences between the analytic and holistic individuals cut across the three common factors of verbal comprehension (Comprehension), perceptual organization (Block Design), and memory (Arithmetic Reasoning) (see e.g., Anastasi, 1982). They also cut across the two major subscales of Performance and Verbal IQ. Such findings are more consistent with the idea of a general ability factor than with any of the typically observed specialized skills. On the other hand, analyses did reveal that the analytic individuals outperformed the holistic ones on a composite score for the memory factor but not for the verbal comprehension factor. The differ- 
ence on the perceptual organization composite score was nearly significant. Such results are more indicative of specialized abilities than of overall intelligence.

Two other findings weigh against the idea of differences in overall ability. The first is the failure to find a difference between the analytic and holistic groups on the Raven's test. The second is J. Smith and Baron's (1981) failure to find SAT score differences between analytic and holistic responders.

If there is a specific ability difference between analytic and holistic responders, it may be that the developmental differences often observed in classification behavior (e.g., Shepp et al., 1980; L. Smith \& Kemler, 1977) are also related to that specific ability. That is, the tendency for individuals to shift from overall similarity to dimensional identity as a basis for classifying objects may be linked to developments in specialized domains rather than to cognitive development in general. Likewise, the tendency for retarded individuals to make more similarity classifications and fewer dimensional identity classifications than their nonretarded peers (see e.g., Burns, 1986; Kemler, 1982) may be a function of deficits in particular skills rather than overall differences in cognitive ability.

The suggestion that there may be a specific ability associated with perceptual analysis is certainly not new. For example, more than 40 years ago, Thurstone (1944) described several perceptual ability factors which were presumed to be distinct from overall intellectual ability. However, if there is a specific type of ability that underlies the tendency to classify analytically instead of holistically, it is difficult to determine from the present results exactly what form that ability takes. Visual analysis is involved in some of the tests that revealed differences, but not in others. In addition, visual analysis may play a role in performance on subtests that did not reveal a significant difference between analytic and holistic responders (e.g., Object Assembly). Likewise, logical reasoning may be involved in some of the subtests that revealed differences between the groups, but it may also be involved in subtests that did not reveal differences (e.g., Similarities and Picture Arrangement). It appears that examination of analytic and holistic individuals on a variety of ability measures may be required before a complete picture of the exact set of skills that differentiates the groups will emerge.

It should also be noted that the present study was concerned specifically with modes of classification in a visual task. This was intentional, since a wide range of findings across several years of investigation has revealed two highly consistent modes of classification in such tasks. However, there may be other aspects of analytic as opposed to holistic processing that are revealed in verbal tasks, and those may indeed relate more strongly to the verbal comprehension measures of intelligence.

Another question that arises in interpreting the present results is whether there is a causal link between ability as measured by intelligence tests and the tendency for individuals to exhibit one or another mode of categorization. In its most extreme form, the link could indicate that individuals with low abilities are unable to analyze the materials into their component dimensions and thus produce responses indicative of integral perception. There is, however, a considerable amount of evidence to suggest that such an interpretation is too extreme. For example, even very young children who normally produce overall similarity groupings for a given set of materials are capable of making dimensional identity comparisons for the same materials if asked to do so (see e.g., Ward, 1980). The holistic individuals in the present study were all college students who scored above average on most of the subtests. Thus it seems unreasonable to argue that such individuals are incapable of decomposing the classification stimuli into component dimensions such as size and angle of orientation of a radius of a circle.

As a less extreme interpretation of the link between ability and mode of classification, it is possible that individuals with higher measured abilities can more readily decompose stimuli into their component dimensions than individuals with lower measured abilities. Since such stimulus decomposition appears to take more time than does generating responses to the whole stimulus (see e.g., J. Smith \& Kemler Nelson, 1984; Ward, 1983), it is possible that individuals with higher abilities complete the process faster and thus generate more dimensional identity responses in a given period of time. The subjects were given $5 \mathrm{sec}$ per triad in the present prescreening task, and thus it is possible that the analytic individuals had enough time to complete the stimulus decomposition process, whereas the holistic individuals did not.

Finally, there may be no causal link between measured ability and categorization behavior at all. It is possible that more intelligent or more highly skilled individuals simply prefer to make dimensional identity classifications. This interpretation would fit with a recent interpretation of the developmental classification findings suggested by L. Smith \& Evans (in press). In their view, the major change that occurs with age is in the extent to which individuals value identity matches. Presumably, adults place a higher value on identity than do young children. In a related way, the present results are consistent with the idea that valuing of identity may be associated with either higher levels of general intelligence or at least with higher levels of particular intellectual skills.

\section{REFERENCES}

Anastasi, A. (1982). Psychological testing. New York: Macmillan. BURNS, B. (1986). Relationship of perceived stimulus structure and intelligence: Further tests of a separability hypothesis. American Journal of Mental Deficiency, 91, 196-200.

COOPER, L. A. (1976). Individual differences in visual comparison processes. Perception \& Psychophysics, 19, 433-444.

COOPER, L. A., \& PoDgorny, P. (1976). Mental transformations and 
visual comparison processes: Effects of complexity and similarity. Journal of Experimental Psychology: Human Perception \& Performance, 2, 503-514.

GARNER, W. E. (1974). The processing of information and structure. Potomac, MD: Erlbaum.

KemLer, D. G. (1982). Classification in young and retarded children: The primacy of overall similarity relations. Child Development, 53, 768-779.

Kogan, N. (1973). Creativity and cognitive style: A life span perspective. In P. Baltes \& K. W. Schaie (Eds.), Life span developmental psychology: Personality and socialization (pp. 145-178). New York: Academic Press.

LEZAK, M. D. (1983). Neuropsychological assessment (2nd ed.). New York: Oxford University Press.

Raven, J. C., Court, J.H., \& Raven, J. (1983). Manual for Raven's Progressive Matrices and Vocabulary Scales. London: Lewis.

Shepp, B. E., Burns, B., \& McDonough, D. (1980). The relation of stimulus structure to perceptual and cognitive development: Further tests of a separability hypothesis. In F. Wilkening, J. Becker, \& T. Trabasso (Eds.), Information integration by children (pp. 113145). Hillsdale, NJ: Erlbaum.

Smith, J. D., BAron, J. (1981). Individual differences in the classification of stimuli by dimensions. Journal of Experimental Psychology: Human Perception \& Performance, 7, 1132-1145.

Smith, J. D., \& Kemler Nelson, D. G. (1984). Overall similarity in adults' classification: The child in all of us. Journal of Experimental Psychology: General, 113, 137-159.

SMITH, L. B., Evans, P. (in press). Similarity, identity and dimensions: Perceptual classification in children and adults. In B. E. Shepp
\& S. Ballesteros (Eds.), Object perception: Structure and process. Hillsdale, NJ: Erlbaum.

Smith, L. B., \& KEMLER, D. G. (1977). Developmental trends in free classification: Evidence for a new conceptualization of perceptual development. Journal of Experimental Child Psychology, 24, 279-298.

Thurstone, L. L. (1944). A factorial study of perception. Chicago, IL: University of Chicago Press.

WARD, T. B. (1980). Separable and integral responding by children and adults to the dimensions of length and density. Child Development, 51, 676-684.

WARD, T. B. (1983). Response tempo and separable-integral responding: Evidence for an integral-to-separable processing sequence in visual perception. Journal of Experimental Psychology: Human Perception \& Performance, 9, 103-112.

WARD, T. B. (1985). Individual differences in processing stimulus dimensions: Relation to selective processing abilities. Perception \& Psychophysics, 37, 471-482.

WARD, T. B., FoleY, C. M., \& ColE, J. (1986). Classifying multidimensional stimuli: Stimulus, task and observer factors. Journal of Experimental Psychology: Human Perception \& Performance, 12, 211-225.

WECHSLER, D. (1981). Manual for the Wechsler Adult Intelligence ScaleRevised. New York: Psychological Corporation.

(Manuscript received April 6, 1988; revision accepted for publication June 24,1988 .) 